\title{
Registration of operant responses in fish ${ }^{1}$
}

\author{
R. E. DAVIS and R. B. KENYON, ${ }^{2}$ \\ UNIVERSITY OF MICHIGAN, Ann \\ Arbor, Michigan 48104
}

A new microminiature inertial switch offers an inexpensive versatile alternative to the crystal phonocartridge as a response-detection device. An operandum for fish based on the inertial switch is described. Results obtained with goldfish suggest that the inertial switch follows response rates up to $2 /$ second; faster rates are confounded by switch contact bounce.

The crystal phonocartridge has had common use in the registration of operant responses in various animals since its application in investigations of fish was first described (see Bitterman, 1966). The object to be struck or operated by the fish is typically fixed to the tip of a stiff rod inserted into the needle holder of the cartridge. Coupled with a high-gain amplifier, an inexpensive crystal can detect very low pressure contacts made by small fish. The crystal response is highly directional, but the target can be positioned against a wall or in a shroud so that the fish is constrained to operate the switch from a sensitive angle. A more important consideration is that an amplifier is needed for each and every phonocartridge in the laboratory, greatly increasing the expense and complexity of a response detector unit. The purpose of this report is to describe a new response device. which has several advantages over the phonocartridge, for investigations of operant responding in fish. The device is based on a microminiature inertial switch of the Gibbs Manufacturing Corp., Janesville, Wisconsin 53545 (cost, approximately $\$ 9$ ).

The Gibbs switch is very sensitive to percussive contacts from all directions. It is very small, easily waterproofed, and it can be placed anywhere in any position in the experimental tank. Also, no amplifier is needed. At least several Gibbs switches can be operated for the cost of a single phonocartridge switch-amplifier system. The main disadvantage of the Gibbs switch is that the contacts bounce for 100 to $400 \mathrm{msec}$. It may thus be unsuitable for response rates exceeding $2 /$ second.

The Gibbs switch consists of a gold-plated TO-18 enclosure can, which is approximately $5 \mathrm{~mm}$ in diam and $4 \mathrm{~mm}$ high, with $10-\mathrm{mm}$ leads. One lead is from a contact mounted on a tiny spring in the center of the can, the other is connected to the wall of the can. Acceleration of the can in any direction deflects the center contact to the wall, momentarily closing the switch. The contacts are rated up to $10 \mathrm{~mA}$ dc at $5 \mathrm{~V} \mathrm{dc}$. We use Model 605 Type I, which is nominally sensitive to forces of $1.5 \mathrm{G}$ (range approximately $1.0-3.0 \mathrm{G}$ ). Figure 1a illustrates an operandum in which a Gibbs is fixed on a $17-\mathrm{cm}$ steel rod which is $0.15 \mathrm{~cm}$ in diam. The leads are extended with insulated wire and then covered with a drop of waterproof cement. The rod and the connecting wires are encased with a short piece of plastic tubing pushed up to the base of the can, into the unhardened cement, to obtain a watertight seal. The can is then covered with a film of epoxy cement insulating the circuit from the water. The other end of the rod is pushed through a tight-fitting hole in a rubber stopper. The completed operandum is held with the stopper in an ordinary chemical clamp that can easily be put in different positions over the surface of the water.

We register contact closures with a BRS-Foringer DigiBit system, as shown in Fig. 1b. Figure 2 is a schematic of an alternative circuit reporting contact closures of the Gibbs switch with a $24-\mathrm{V} \mathrm{dc}$ relay. The circuit is based on a relay driver module of Texas Instruments Incorporated (P.O. Box 5012, M.S. 308, Dallas, Texas 75222) costing approximately $\$ 1.25$. The maximum response frequency of the Gibbs is limited with a oneshot to block the registration of postresponse contact bounces or "false responses," which would otherwise inflate the response rate. III investigations with 7 - to $12-\mathrm{cm}$ goldfish, for example, we set the response oneshot at $400 \mathrm{msec}$. The contact bounces that follow a unitary hit by a fish seldom last longer than $300 \mathrm{msec}$ and rarely exceed $400 \mathrm{msec}$. But goldfish sometimes mouth the tip of the manipulandum for an instant or flick it rapidly with their protrusible lips. Either of these responses produces a complex train of contact bounces, several of which may register as responses spaced 400 msec apart. Thus, while the 400-msec oneshot virtually eliminates the registration of false responses, an unknown number of high-frequency responses are also filtered out and still others are registered, but with a distorted temporal distribution. A phonocartridge switch (Astatic N6-2) that we occasionally use in place of the Gibbs shows less "bounce"; a 200-msec oneshot, for example, seems to block registration of most postresponse oscillations in the output of the crystal.

The marked effect on recorded response rates of varying the duration of the response oneshot a few 10 ths of a second can be seen in Table 1. A Gibbs and a phonocartridge switch were compared to establish how their performance as operandum switches differs with various oneshot durations. The data were obtained with three $7-\mathrm{cm}$ goldfish maintained on a variable interval 3-min schedule of food reinforcement for six daily 30-min sessions. The Ss were selected specifically for their history of high stable response rates (10 to $12 /$ minute). The food reinforcement consisted of a few brine shrimp automatically dispensed in a drop of water next to the operandum (Davis \& Hirtzel, 1970). The tank was a 9.5-1 aquarium, $15 \times 30 \times 18 \mathrm{~cm}$ deep, filled to a depth of $15 \mathrm{~cm}$ with a continuous flow of dechlorinated $20^{\circ} \mathrm{C}$ water. The operandum was held vertically over the center of the tank so that the tip was $2 \mathrm{~cm}$ beneath the surface of the water. The Gibbs was used as the operandum switch on Days 1,3, and 5 of the experiment, and the phonocartridge was used on Days 2, 4, and 6 . The operandum for the phonocartridge was a TO-18 can, which was mounted on a steel rod closely resembling the Gibbs. In each daily session, responses registered with a $400-\mathrm{msec}$ oneshot and, simultaneously, with a shorter test oneshot. The test oneshot was $100 \mathrm{msec}$ on Days 1 and 2, $200 \mathrm{msec}$ on Days 3 and 4, and $300 \mathrm{msec}$ on Days 5 and 6. Thus. in successive daily sessions each of the three test oneshots was paired first with the Gibbs and then with the phonocartridge. The oneshot outputs were

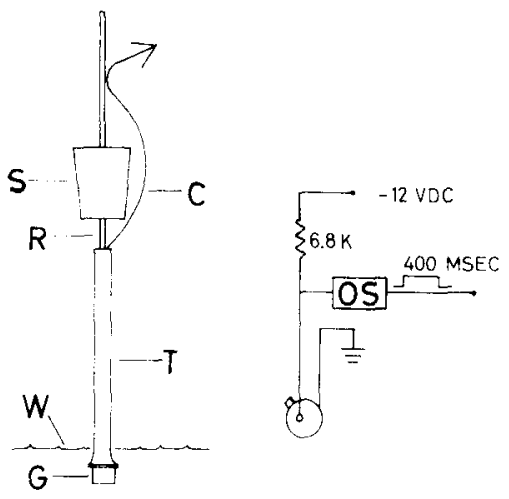

Fig. 1. (a) The operandum diagrammed on the left consists of a Gibbs inertial switch in a TO-18 can (G), a plastic tube (T) covering two connecting wires (C) and a supporting steel rod $(\mathrm{R})$, which is pushed through a subber stopper (S). In practice, the tip of the operandum is positioned about $2 \mathrm{~cm}$ below the water surface (W). (b) The schematic on the right is of the oneshot circuit used to register contact closures of the Gibbs. 


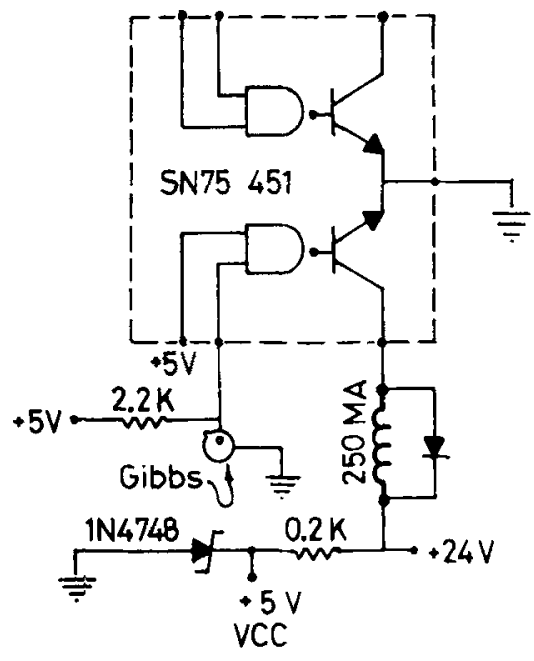

Fig. 2. A circuit to report contact closures of a Gibbs switch with a 24-V dc relay. The SN75451 Dual Relay Driver module, Texas Instruments, Inc., will operate two independer: Gibbs switches. One switch and its relay are shown attached to the integrated circuit; to add the second, the $+5-\mathrm{V}$ cc obtained with the illustrated Zener diode circuit is simply extended to the other driver.

tallied on independent Gerbrands cumulative recorders.

The results showed that with the 400 and $300-\mathrm{msec}$ oneshots the Gibbs was as effective in detecting responses as was the phonocartridge. The $400-\mathrm{msec}$ oneshot registered an average of 372 responses in the three sessions with the Gibbs and 377 responses in the phonocartridge sessions. The $300-\mathrm{msec}$ oneshot registered only a few more responses than the 400 msec oneshot, and the increase was statistically equivalent for the two switches. A two-way analysis of variance of the data in Table 1 reveals a significant switch effect $(F=117$, $\mathrm{df}=1 / 4, \quad \mathrm{p}<.001), \quad \mathrm{a}$ oneshot effect $(\mathrm{F}=78, \quad \mathrm{df}=2 / 2, \quad \mathrm{p}<.01) \quad$ and $\mathrm{a}$ oneshot-switch interaction $\quad(F=10$, $\mathrm{df}=2 / 4, \mathrm{p}<.05$ ). The present results do not indicate exactly how many of the responses tallied by the various test

Table 1

Percent Increase in Registered Responses with One-Shots Shorter Than $400 \mathrm{Msec}$

\begin{tabular}{lllcl}
\hline & & \multicolumn{3}{c}{ One-Shot Duration } \\
\cline { 3 - 5 } Switch & Subject & 300 & 200 & 100 \\
\hline Gibbs & $\mathrm{N} 28$ & 4.9 & 44.4 & 56.1 \\
& $\mathrm{~N} 42$ & 5.1 & 38.2 & 78.0 \\
& $\mathrm{~N} 43$ & 9.4 & 52.1 & 69.7 \\
& & $6.5 \pm 1.5^{*}$ & $44.9 \pm 4.0$ & $68.0 \pm 6.4$ \\
Phonocartridge & $\mathrm{N} 28$ & 4.9 & 9.0 & 21.8 \\
& $\mathrm{~N} 42$ & 3.5 & 11.6 & 24.3 \\
& $\mathrm{~N} 43$ & 1.9 & 8.4 & 26.9 \\
& & $3.4 \pm 1.5$ & $9.7 \pm 1.7$ & $24.27 \pm 2.52$ \\
\hline
\end{tabular}

$* \bar{X} \pm S D$

oneshots are false, or represent a contact bounce produced by a prior response. But we suggest that the differences between the two switches obtained with the 100 and 200 -msec oneshots are largely owed to the long-lasting contact bounces of the Gibbs. Some of the phonocartridge responses detected with the $100-\mathrm{msec}$ oneshot are also false; direct observations showed that solid unitary hits were occasionally tallied as two responses. The between-Ss differences in responses on the Gibbs further suggest that the frequency of false responses registration varies with the individual. Conceivably, differences in the force of a hit, if not the direction, are reflected in the duration of the train of contact bounces.

The Gibbs can register hits when held in any position in the tank. For example, a Gibbs attached to a short flexible rod, or a strip of plastic, can be used as an operandum that projects from the bottom or through a hole in a vertical or horizontal partition. The connecting wires are simply sealed from the water in an ordinary plastic air hose. Hits on an illuminated, transparent, or translucent target are easily registered. It is unnecessary to support the target from above, as would be required for a phonocartridge switch. The target can be held in an opening in an opaque panel with a strip of plastic as a hinge, and the Gibbs can be cemented directly onto the target or a rearward projection on the target completely hidden from the S's view. If two or more targets are to operate in a tank, each Gibbs must be protected from substrate vibrations produced by hits on the others. Also, targets much larger in outline than the Gibbs enclosure may need to be shielded from water currents produced by fish.

The Gibbs inertial switch clearly has many possible applications in behavioral and physiological research. Its simple make-break action, high sensitivity, small size, and low cost more than offset the disadvantage of the prolonged contact bounce. In addition, 6 months of daily operation has revealed no drift in sensitivity, and no maintenance has been required, a performance that is unmatched by any of the phonocartridge-amplifier systems that we have used.

\section{REFERENCES}

BITTERMAN, M. E. Animal leaming. In J. 8. Sidowski (Ed.), Experimental methods and instrumentation in psychology. New York: McGraw-Hill, 1966. Pp. 451-484.

DAVIS, R. E., \& HIRTZEL, M. S. An inexpensive brine shrimp dispenser for fish. Behavior Research Methods \& Instrumentation, $1970,2,262$.

\section{NOTES}

1. Supported by NIMH Research Development Award K2-MH-22,183 (R.E.D.). Reprints may be obtained from R.E. Davis, Mental Health Research Institute, University of Michigan, Ann Arbor, Michigan 48104.

2. We wish to thank our colleagues James Mullison and Kenneth Báchman for their electronic expertise and patient assistance. 